\title{
Critical number of atoms for attractive Bose-Einstein condensates with cylindrically symmetrical traps
}

\author{
A. Gammal, ${ }^{1}$ T. Frederico, ${ }^{2}$ and Lauro Tomio ${ }^{1}$ \\ ${ }^{1}$ Instituto de Física Teórica, Universidade Estadual Paulista, 01405-900 São Paulo, Brazil \\ ${ }^{2}$ Departamento de Física, Instituto Tecnológico da Aeronáutica, Centro Técnico Aeroespacial, \\ 12228-900 São José dos Campos, São Paulo, Brazil
}

(Received 12 April 2001; published 10 October 2001)

\begin{abstract}
We calculated, within the Gross-Pitaevskii formalism, the critical number of atoms for Bose-Einstein condensates with two-body attractive interactions in cylindrical traps with different frequency ratios. In particular, by using the trap geometries considered by Roberts et al. [Phys. Rev. Lett. 86, 4211 (2001)], we show that the theoretical maximum critical numbers are given approximately by $N_{c}=0.55\left(l_{0} /|a|\right)$. Our results also show that, by exchanging the frequencies $\omega_{z}$ and $\omega_{\rho}$, the geometry with $\omega_{\rho}<\omega_{z}$ favors the condensation of larger number of particles. We also simulate the time evolution of the condensate when changing the ground state from $a=0$ to $a<0$ using a $200 \mathrm{~ms}$ ramp. A conjecture on higher-order nonlinear effects is also added in our analysis with an experimental proposal to determine its signal and strength.
\end{abstract}

DOI: 10.1103/PhysRevA.64.055602

PACS number(s): 03.75.Fi, 32.80.Pj, 11.10.Lm, 02.60.Lj

Bose-Einstein condensates (BECs) with attractive interactions have been realized with ${ }^{7} \mathrm{Li}$ since 1995 by Bradley et al. [1] culminating with experiments that have direct observation of the growth and collapse of this condensate [2]. Measurements of the maximum critical number of atoms $N_{c}$ in the condensate, in a trap almost spherical, were in good agreement with the theoretical predicted numbers, within the experimental uncertainties.

Recently Bose-Einstein condensation has been achieved with ${ }^{85} \mathrm{Rb}$ [3] by means of Feshbach resonance, which allowed wide tunning of the scattering length $a$ from negative to positive. The ability to control the scattering length is used to control and measure the stability condition with the corresponding critical number of atoms.

In Ref. [4], it was first shown numerically that for attractive interactions (negative scattering length $a$ ) the system becomes unstable if a maximum critical number of atoms $N_{c}$ is achieved. This limit can be stated in a convenient expression by

$$
\frac{N_{c}|a|}{\sqrt{(\hbar / m \omega)}}=k
$$

where $m$ is the mass of the particle confined in a trap with frequency $\omega$ and $k$ is a dimensionless constant, directly associated with the critical number of atoms $N_{c}$. So, by using the above assumption of a spherically symmetrical trap, several authors [5,6], including us [7], have calculated $k$ with a variety of methods. With the precision given in Ref. [7], $k$ $=0.5746$. In Ref. [8], the critical number was calculated for a nonsymmetrical geometry, but in a case that the frequency ratio is not too far from the unity $\left(\omega_{z} / \omega_{\rho}=0.72\right)$, giving a result for the number of atoms almost equal to the spherical one.

One may also infer from the variational treatment used in Ref. [9] that the constant $k$ depends on the symmetry of the trap. Variational estimates were also considered in Ref. [10]. So, in cases of nonspherical symmetry, the number $k$ will be dependent on the ratios of the trap frequencies, with the equation being scaled by some averaged frequency. As in most of the cases considered experimentally, the spatial symmetry is almost cylindrical, with the trap frequencies given by $\omega_{x} \approx \omega_{y}$ and $\omega_{z}$, we assume $\omega_{\rho}=\omega_{x}=\omega_{y}$ and a geometrical averaged frequency given by $\bar{\omega}=\left(\omega_{z} \omega_{\rho}^{2}\right)^{1 / 3}$.

We define

$$
\lambda \equiv \frac{\omega_{z}}{\omega_{\rho}},
$$

such that the trap will have a "pancake-shape" if $\lambda>1$; and a "cigar-shape" if $\lambda<1$. The spherical symmetry is recovered with $\lambda=1$. It is convenient to redefine the number $k$ given in Eq. (1), showing explicitly its dependence on $\lambda$. In this case, the critical number of atoms $N_{c}$ is given by

$$
N_{c}\left(\omega_{\rho}, \omega_{z}\right)=\frac{k(\lambda)}{|a|} l_{0}=\lambda^{-1 / 6} \frac{k(\lambda)}{|a|} l_{\rho}=\lambda^{1 / 3} \frac{k(\lambda)}{|a|} l_{z}
$$

where $l_{0} \equiv \sqrt{\hbar / m \bar{\omega}}, l_{\rho} \equiv \sqrt{\hbar / m \omega_{\rho}}$ and $l_{z} \equiv \sqrt{\hbar / m \omega_{z}}$.

Here, in Eq. (3), we observe explicitly the dependence of $N_{c}$ in relation to $\lambda$. By exchanging the frequencies $\omega_{\rho}$ and $\omega_{z}$ in the trap, we observe that $l_{\rho} \rightarrow l_{z}, l_{z} \rightarrow l_{\rho}$ and $\lambda \rightarrow 1 / \lambda$. The exchange ratio in this case is given by

$$
R(\lambda) \equiv \frac{N_{c}\left(\omega_{\rho}, \omega_{z}\right)}{N_{c}\left(\omega_{z}, \omega_{\rho}\right)}=\lambda^{1 / 6} \frac{k(\lambda)}{k(1 / \lambda)} .
$$

$R(\lambda)$ is the relevant factor that affects the number of particles in the condensate, when exchanging the frequencies in a cylindrical configuration. In the case that $k(\lambda) \sim k(1 / \lambda)$, we may conclude that $\omega_{z}>\omega_{\rho}$ results in a larger number of particles inside the trap in the critical limit.

The above considerations and the numerical calculations of $k(\lambda)$ that we are communicating are relevant to be taken into account in experiments with BEC in cylindrical traps with negative $a$, such as the experiments that have been per- 
formed in JILA with ${ }^{85} \mathrm{Rb}$. Roberts et al. (JILA) have used a "cigar-shape" symmetry in their experiment [3,11]. They have determined, recently, that $k=0.459 \pm 0.012$ (statistical) \pm 0.054 (systematic), for a nonspherical trap, where the frequencies were $17.24 \times 17.47 \times 6.80 \mathrm{~Hz}$. Using the above notation, we may take $\omega_{\rho}=\sqrt{\omega_{x} \omega_{y}}=2 \pi \times 17.35 \mathrm{~Hz}$. So, the corresponding value of $\lambda$ used in Ref. [11] was $\omega_{z} / \omega_{\rho}$ $=6.80 / 17.35=0.3919$.

Since the JILA trap is nonspherical, it is worthwhile to determine numerically the values of $k$, for different $\lambda$. Our main goal in the present report is to systematically calculate $k(\lambda)$ in cylindrical symmetry, either in pancake $(\lambda>1)$ or cigar shape $(\lambda<1)$, in order to verify the favorable geometry of the trap to condensate a larger number of atoms, when the two-body scattering length is negative. As we are going to show, the slight discrepancy found by the JILA group, when comparing their experimental value of $k$ with the theoretical results, may partially be explained by the present report.

For an atomic system with negative scattering length and trapped by an external harmonic oscillator (nonsymmetric, in general), the Bose-Einstein condensate may be described by the Gross-Pitaevskii equation

$$
\begin{aligned}
\mathrm{i} \hbar \frac{\partial}{\partial t} \Psi(\vec{r}, t)= & {\left[-\frac{\hbar^{2}}{2 m} \nabla^{2}+\frac{m}{2}\left(\omega_{x}^{2} x^{2}+\omega_{y}^{2} y^{2}+\omega_{z}^{2} z^{2}\right)\right.} \\
& \left.-\frac{4 \pi \hbar^{2}|a|}{m}|\Psi(\vec{r}, t)|^{2}\right] \Psi(\vec{r}, t) .
\end{aligned}
$$

The conditions for the validity of this formalism to describe atomic systems with negative scattering lengths are given in Ref. [12]. Deviations due to quantum fluctuations and tunneling, that occur near the collapsing region, were studied in Refs. $[6,13]$. As it appears from such studies, the decay probability due to quantum tunneling (that will effectively reduce $N_{c}$ ) is negligible, unless $N \approx N_{c}$.

The wave function, given by

$$
\Psi(\vec{r}, t)=\exp (-\mathrm{i} \mu t / \hbar) \Psi(\vec{r}, 0),
$$

where $\mu$ is the chemical potential, is normalized to the number of atoms:

$$
\int d^{3} r|\Psi(\vec{r}, t)|^{2}=N
$$

Using cylindrical symmetry $\left(\omega_{x}=\omega_{y}=\omega_{\rho}\right)$ and considering dimensionless units $\left[\tau \equiv \bar{\omega} t, \rho^{2} \equiv(2 m \bar{\omega} / \hbar)\left(x^{2}+y^{2}\right), \zeta^{2}\right.$ $\left.=(2 m \bar{\omega} / \hbar) z^{2}\right]$, followed by a scaling of the wave function,

$$
\Phi \equiv \Phi(\rho, \zeta ; \tau) \equiv \sqrt{\frac{4 \pi \hbar|a|}{m \bar{\omega}}} \Psi(\vec{r}, t)
$$

we have

$$
\mathrm{i} \frac{\partial \Phi}{\partial \tau}=\left[-\nabla^{2}+\left(\frac{\omega_{\rho}}{\bar{\omega}}\right)^{2} \frac{\rho^{2}}{4}+\left(\frac{\omega_{z}}{\bar{\omega}}\right)^{2} \frac{\zeta^{2}}{4}-|\Phi|^{2}\right] \Phi,
$$

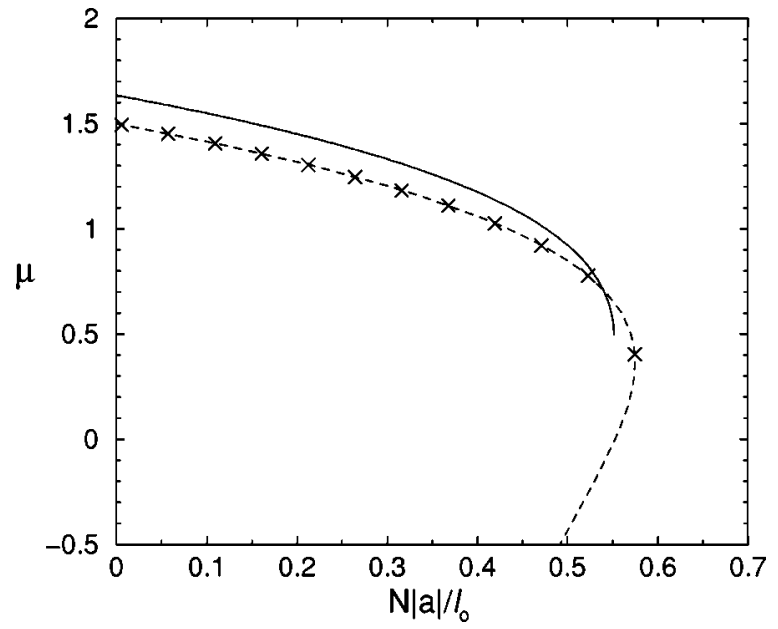

FIG. 1. The chemical potential $\mu$ is given in units of $\hbar \bar{\omega}$, as a function of $N|a| / l_{0}$. Results with spherical symmetry $(\lambda=1)$, in dashed line and with $\times$, are compared with results using $\lambda$ $=6.80 / 17.35$ (solid line). Dashed line was obtained using shootingRunge-Kutta method, while the $x$ and the solid line were obtained by propagation in imaginary time.

$$
\text { where } \quad \nabla^{2} \equiv \frac{1}{\rho} \frac{\partial}{\partial \rho}\left(\rho \frac{\partial}{\partial \rho}\right)+\frac{\partial^{2}}{\partial \zeta^{2}} \text {. }
$$

Given the Eqs. (7) and (8), we obtain the normalization of $\Phi$ to a defined reduced number of atoms $n$

$$
\int_{-\infty}^{\infty} d \zeta \int_{0}^{\infty} d \rho \rho|\Phi|^{2}=4 \sqrt{2} \frac{N|a|}{l_{0}} \equiv 2 n
$$

where, in the critical limit, $n=n_{c}=2 \sqrt{2} k$. Equation (9) depends only on the ratio $\lambda=\left(\omega_{z} / \omega_{\rho}\right)$ :

$$
\beta \Phi=\left[-\nabla^{2}+\lambda^{-(2 / 3)} \frac{\rho^{2}}{4}+\lambda^{(4 / 3)} \frac{\zeta^{2}}{4}-|\Phi|^{2}\right] \Phi,
$$

where $\beta \equiv \mu /(\hbar \bar{\omega})$. So, the normalization constant $n$, given by Eq. (10), as well as $k$, will depend only on $\lambda$.

In our calculation of Eq. (9), we employed the relaxation method propagating in the imaginary time and renormalizing $\Phi$ to $2 n$ at every step $[8,14]$. We searched for stable solutions by varying the number $n$ till a critical limit $n_{c}$. No ground-state solutions are possible for $n>n_{c}$. In Fig. 1, we have the corresponding results for the chemical potential as a function of $N|a| / l_{0}=n /(2 \sqrt{2})$. To obtain the results shown in Fig. 1, we first tested our code by running the symmetrical case $\lambda=1 \quad\left(\omega_{z}=\omega_{\rho}\right)$ and comparing the results with very precise ones that we have previously calculated with the shooting-Runge-Kutta algorithm [7]. The plot with $\times$ marks corresponds to the imaginary time propagation method while the dashed-line plot refers to the shooting-Runge-Kutta method (in both, spherical symmetry was used). One should note that the unstable solutions (back bending branch) are not accessible by the time-dependent method. The plot with solid line shows our results for a cylindrical symmetry, with the JILA parameters given in [11], i.e., $\omega_{z}=2 \pi \times 6.80 \mathrm{~Hz}$ 
TABLE I. Numerical solutions for the critical constant $k$, as a function of $\lambda=\omega_{z} / \omega_{\rho} . k=k_{s}$ is for spherical symmetry. An asterisk indicates the symmetry considered by the JILA group; alternatively; a dagger the corresponding "pancake-shape" symmetry.

\begin{tabular}{lcc}
\hline \hline \multicolumn{1}{c}{$\lambda$} & $k$ & $k / k_{s}$ \\
\hline 0.01 & 0.314 & 0.547 \\
0.02 & 0.352 & 0.613 \\
0.05 & 0.411 & 0.716 \\
0.1 & 0.460 & 0.801 \\
0.2 & 0.509 & 0.886 \\
0.3 & 0.535 & 0.931 \\
$(6.80 / 17.35)^{*}$ & 0.550 & 0.957 \\
0.5 & 0.560 & 0.975 \\
$2 / 3$ & 0.570 & 0.992 \\
1.0 & 0.5746 & 1.000 \\
1.5 & 0.570 & 0.992 \\
2.0 & 0.561 & 0.976 \\
$(17.35 / 6.80)^{\dagger}$ & 0.549 & 0.956 \\
$\sqrt{8}$ & 0.544 & 0.946 \\
3.0 & 0.541 & 0.941 \\
4.0 & 0.518 & 0.902 \\
5.0 & 0.498 & 0.867 \\
10.0 & 0.441 & 0.767 \\
20.0 & 0.376 & 0.655 \\
50.0 & 0.294 & 0.511 \\
\hline \hline
\end{tabular}

$\omega_{\rho}=2 \pi \times 17.35 \mathrm{~Hz}$. In this case, $k=0.550$ is approximately $4 \%$ lower than the spherical case.

For the propagation, we have used the PeacemanRachford alternating-direction implicit method [15]. The time evolution for cylindrical symmetry was performed with a code used in [16]. Our discretization was up to $200 \times 200$ in $\rho$ and $\zeta$ space directions, and up to 50 in the variable $\tau$ $(=\bar{\omega} t)$, with steps of 0.001 . We also considered $\rho_{\max }$ and $\zeta_{\max }$ ranging from 2 to 10 depending on the symmetry. In the extreme nonsymmetric cases $(\lambda$ or $1 / \lambda \gg 1)$, the results are more sensible to the grid spacing and to these maximum values. In these cases, a lack of precision may occur in the third decimal digit of the results shown in Table I.

In Table I, we present the numerical results for the critical constant $k$ as a function of the parameter $\lambda=\omega_{z} / \omega_{\rho}$, which may be useful to analyze experiments with different cylindrical shapes. Clearly, the optimal value for $k$ occurs for spherically symmetric traps $(\lambda=1)$, as one could also infer from the variational calculations given in [9]. In particular, we determined the values of $k$ for the ratios considered in the JILA experiment $[3,11]$ : The theoretical constant, $k \approx 0.55$, is about $4 \%$ lower than the corresponding number with spherical symmetry $\left(k_{s}=0.5746\right)$. This may partially explain the small disagreement observed in Ref. [11] when comparing their result with theoretical ones.

By exchanging the frequencies $\omega_{\rho}$ and $\omega_{z}$ in a cylindrical symmetry, it is also shown that the "pancake-shape" symmetry $\left(\omega_{z}>\omega_{\rho}\right)$ is preferable (in order to obtain a larger $N_{c}$ ) when $k(\lambda) \approx k(1 / \lambda)$. Considering the exchange ratio presented in Eq. (4) and the results shown in Table I for $k(\lambda)$,

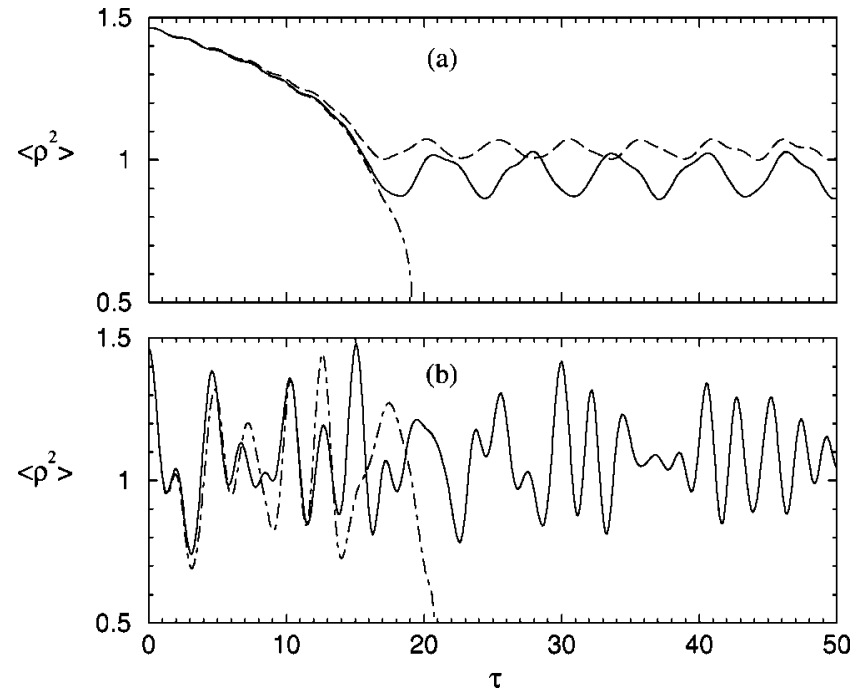

FIG. 2. Time evolution of the dimensionless mean-square radius ( $\rho$ ) of the condensate when changing the ground state from $a=0$ to $a<0$. We have considered a $200 \mathrm{~ms}(\tau=\bar{\omega} t=16)$ linear ramp in (a); and an instantaneously shift in (b). In (a), the dashed, solid, and dot-dashed lines correspond to the ramping until $N|a| / l_{0}=0.9 k_{s}$, $0.94 k_{s}, 0.95 k_{s}$, respectively. In (b), the dashed line corresponds to the ramping until $N|a| / l_{0}=0.9 k_{s}$; and the solid line corresponds to the ramping until $N|a| / l_{0}=0.91 k_{s} . k_{s}$ is the collapse constant $k$ $=N|a| / l_{0}$ in spherical symmetry. Trap parameters were $\omega_{\rho}=2 \pi$ $\times 17.35 \mathrm{~Hz}$ and $\omega_{z}=2 \pi \times 6.80 \mathrm{~Hz}$.

one may verify the optimal geometry to increase the critical number of atoms $N_{c}$ trapped in a condensate. Analyzing the "pancake-shape symmetry," related with the "cigar-shape symmetry" considered by the JILA group in Ref. [11], we note that $\lambda_{1}=17.35 / 6.80=2.5517$, and $\lambda_{2}=1 / \lambda_{1}=0.3919$. As shown in Table I, both cases will give us practically the same constant number $k \approx 0.55$. So, the relevant factor that will decide the convenient symmetry to condensate a larger number of atoms is given by Eq. (4), in this case; and this favors the "pancake-shape" geometry

$$
R\left(\lambda_{1}=17.35 / 6.80\right) \approx 1.17 \text {. }
$$

The number of atoms in the condensate may be increased by a factor of $\sim 17 \%$, just by exchanging the geometry of the trap. The above factor may be verified experimentally, as well as other frequency ratios, with the help of Table I and the present relations given for $k(\lambda)$ and $R(\lambda)$.

We should add that the other part of the observed discrepancy in the experimental value of $k$ could be explained by an early collapse of the condensate due to a dynamical chirp in the wave function when moving the system from $a>0$ to $a$ $<0$. It means that when changing the scattering length from a positive to a negative value, the energy minimum with $a$ $>0$ is greater than the corresponding energy minimum with $a<0$, such that the system will collapse at a lower critical number [17].

We simulated the realistic situation with the parameters given in Ref. [11]. We depart from the ground state with $a$ $=0$ and then ramp it to $a<0$ in 200 ms. In Fig. 2(a), we 
show the time evolution of the mean-square radius $\rho$ for different final negative scattering lengths. For a final value of $N|a| / l_{0}$ lower or equal to $0.94 k_{s}$, the system presents collective excitations; for a larger value, the system collapses. So, we conclude that the dynamical effects may only account for about $2 \%$ of the discrepancy observed between the experimental and theoretical values of $k$. This result implies that the total correction due to the nonspherical symmetrical trap and due to dynamical effects may only account for a diminishing of about $6 \%$ in the spherical predicted value of $k$. For comparison, we also present in Fig. 2(b) the corresponding instantaneous shift from $a=0$ to $a<0$.

A larger deviation of $k$ is expected in this case, as this numerical simulation [shown in Fig. 2(b)] corresponds to a larger chirp in the wave function than in the case that $a$ is "ramping" slowly in time. We found that at $N|a| / l_{0}$ $=0.9 k_{s}$, the system has complex higher mode nonlinear oscillations; for a larger value of $N|a| / l_{0}$, it collapses. So, even in this case, we may account to a maximum of $10 \%$ shift in the value of $k$ (including dynamical and nonspherical effects), when comparing with the spherical result.

As temperature dependence is being ruled out in the experimental analysis, another interesting possibility, which could explain a larger deviation in the value of the constant $k$, may be attributed to higher-order nonlinear effects, which in this case, are contributing to increase the attractive part of the effective nonlinear potential. The relevant effect of a real three-body effective interaction, given by a quintic term $g_{3}|\Phi|^{4} \Phi$ in the right-hand side (r.h.s.) of Eq. (11), was already pointed out in [18]. If $g_{3}$ is positive, there is a possibility of two phases in the condensate [18]. However, in the case where $g_{3}$ has the same negative sign as the two-body interaction, one may also obtain a relevant contribution that may explain a smaller value for the constant $k$, as it is occurring in the present case. In order to obtain the missing part of deviation $(\sim 10-15 \%)$, we estimated numerically that it is enough to have $g_{3} \approx-0.03$.

A way to obtain some definitive conclusion about the above conjecture of a relevant role of higher-order nonlinearity, is open experimentally by examining particularly the case $a \approx 0$, when the cubic term in the r.h.s. of Eq. (11) is replaced by a quintic term. A limit in the number of particles at this particular value of $a$ is a good indication of negative higher-order nonlinearity; and, given $N_{c}$, the corresponding strength of the nonlinear interaction (which should mainly come from three-body effects) can be estimated.

We would like to thank Randall Hulet and Arjendu Pattanayak for useful discussions. This work was partially supported by Fundação de Amparo à Pesquisa do Estado de São Paulo and Conselho Nacional de Desenvolvimento Científico e Tecnológico.
[1] C.C. Bradley, C.A. Sackett, J.J. Tollett, and R.G. Hulet, Phys. Rev. Lett. 75, 1687 (1995); C.C. Bradley, C.A. Sackett, and R.G. Hulet, ibid. 78, 985 (1997).

[2] J.M. Gerton, D. Strekalov, I. Prodan, and R.G. Hulet, Nature (London) 408, 692 (2000).

[3] S.L. Cornish, N.R. Claussen, J.L. Roberts, E.A. Cornell, and C.E. Wieman, Phys. Rev. Lett. 85, 1795 (2000).

[4] P.A. Ruprecht, M.J. Holland, K. Burnett, and M. Edwards, Phys. Rev. A 51, 4704 (1995).

[5] M. Houbiers and H.T.C. Stoof, Phys. Rev. A 54, 5055 (1996); R.J. Dodd et al., 54, 661 (1996); N. Akhmediev, M.P. Das, and A.V. Vagov, Int. J. Mod. Phys. B 13, 625 (1999); L. Bergé, T.J. Alexander, and Y.S. Kivshar, Phys. Rev. A 62, 023607 (2000).

[6] C. Huepe, S. Métens, G. Dewel, P. Borckmans, and M.E. Brachet, Phys. Rev. Lett. 82, 1616 (1999).

[7] A. Gammal, T. Frederico, and L. Tomio, Phys. Rev. E 60, 2421 (1999).

[8] F. Dalfovo and S. Stringari, Phys. Rev. A 53, 2477 (1996).

[9] C.A. Sackett, C.C. Bradley, M. Welling, and R.G. Hulet, Appl. Phys. B: Lasers Opt. 65, 433 (1997).
[10] M. Wadati and T. Tsurumi, J. Phys. Soc. Jpn. 68, 3840 (1999).

[11] J.L. Roberts, N.R. Claussen, S.L. Cornish, E.A. Donley, E.A. Cornell, and C.E. Wieman, Phys. Rev. Lett. 86, 4211 (2001).

[12] F. Dalfovo, S. Giorgini, L.P. Pitaevskii, and S. Stringari, Rev. Mod. Phys. 71, 463 (1999).

[13] H.T.C. Stoof, J. Stat. Phys. 87, 1353 (1997); M. Ueda and K. Huang, Phys. Rev. A 60, 3317 (1999).

[14] M.J. Holland, D.S. Jin, M.L. Chiofalo, and J. Cooper, Phys. Rev. Lett. 78, 3801 (1997).

[15] D.W. Peaceman and H.H. Rachford Jr., J. Soc. Ind. Appl. Math. 3, 28 (1955). See, also, C. F. Gerald and P. O. Wheatley, Applied Numerical Analysis (Addison-Wesley, New York, 1998).

[16] A.K. Pattanayak, A. Gammal, C.A. Sackett, and R.G. Hulet, Phys. Rev. A 63, 033604 (2001).

[17] F.K. Abdullaev, A. Gammal, L. Tomio, and T. Frederico, Phys. Rev. A 63, 043604 (2001).

[18] A. Gammal, T. Frederico, L. Tomio, and Ph. Chomaz, Phys. Rev. A 61, 051602(R) (2000). 